\title{
CORRUPCIÓN Y DESARROLLO EN JUNIN. UN ENFOQUE MICROECONOMICO
}

\author{
CORRUPTION AND DEVELOPMENT IN JUNIN. A MICRO-ECONOMIC FOCUS
}

\author{
Saúl Darío Díaz Maravi', Manuel Larrauri Rojas'
}

\section{RESUMEN}

El trabajo analizó desde los agentes económicos, el nivel de corrupción que incide de manera directa en el proceso de desarrollo. Los objetivos fueron: Determinar en que medida los agentes económicos institucionales se encuentran en corrupción y como afecta al proceso de desarrollo, luego precisar cuales son los sectores más corruptos a nivel departamental. Es decir, cuanto más corrupto es una sociedad, mas atrasado se encuentra y esto se puede ver a nivel mundial, donde se puede observar que los países más pobres son los más corruptos. La hipótesis central fue que el departamento de Junín se encuentra con altos niveles de corrupción. En esta investigación descubrimos que no importa la ideología o el nivel de ingresos para que una persona se considere o cometa actos corruptos. Igual sin distinción de ideología o nivel de ingresos las personas son corruptas y más responde a un proceso cultural. Pero al mismo tiempo los sectores de mayores ingresos obtienen mayores beneficios en situaciones de alta corrupción como en el caso peruano y en el departamento de Junín. Y simultáneamente con estos niveles de corrupción aumenta la pobreza y se frena el proceso de desarrollo, por lo que se demostró la hipótesis encontrando que a Junín se le considera como "la capital de la corrupción" con un 12,5\% de percepción de corrupción y gestión gubernamental, que representa el doble del promedio nacional.

Palabras Clave: Corrupción, desarrollo, agentes económicos, Junín.

\begin{abstract}
This work analyzed economic agents, corruption level in Junin department, and the impacts in a direct way in the development process. The objectives were: To determinate the level corruption about in the institutional economic agents. I can say, the more corrupt it is a society, but late he/she is and this one can see at world level, where one can observe that the poorest countries are the most corrupt. Our central hypothesis was that the department of Junin is in high levels of corruption and this hypothesis it was contrasted, finding that to Junín he/she is considered as "the capital of the corruption" $(12,5 \%$ of index of perception of corruption and government administration, double the national average). Our hypothesis was also that the judicial power and the professionals linked to the judicial power, like they are the lawyers, they were the sectors more corrupt and this hypothesis was contrasted affirmatively. In this investigation process we discoved that it does care the ideology or the level of revenues so that a person is considered or make corrupt acts. Same without ideology distinction or level of revenues people are corrupt and more responds to a cultural process. But at the same time the sectors of more revenues obtain bigger benefits in situations of high corruption like in the Peruvian case and in the department of Junín. And simultaneously with these levels of corruption the poverty increases and the development process is broken.
\end{abstract}

Key words: Corruption, development, economics people, Junín. 


\section{INTRODUCCIÓN}

En el proceso de corrupción seguido en los últimos treintas años en el Perú, éste se ha agravado, por tal motivo nos interesó analizar la corrupción en el departamento de Junín, desde la perspectiva de los agentes microeconómicos. Ya que cualquier acto por más trivial que sea tiene un componente cultural y un componente económico. Un hecho de corrupción tiene esos dos componentes: cultural y económico principalmente. Lamentablemente encontramos que la corrupción en nuestro departamento y en nuestro país tiene más del aspecto cultural y que se remonta a la época prehispánica (2), añadiendo elementos de la de la cosmovisión occidental.

De tal simbiosis surgió la corrupción muy a la "peruana" que se puede traducir en el "comportamiento criollo" alabada por muchos, pero que desgraciadamente es la fuente y origen de la corrupción que incluso se da nivel lingüístico; ya que la corrupción comienza con la distorsión innecesaria de las palabras con las que nos comunicamos, es decir, el uso de la replana, más aún siendo el idioma castellano uno de los más ricos en palabras es absurdo utilizar el lenguaje vulgar de la replana o barbarismos.

Entonces las personas que están afectas al uso excesivo de este tipo de lenguaje son porque también en sus actos cotidianos y en sus sentimientos no son sinceros, sino buscan de manera casi inconsciente distorsionar la realidad en provecho suyo, siempre con la consigna de sacar beneficio de todo y ante todo. Luego cuando se pasa a ejercer algún poder entonces comienza la espiral de corrupción que tiene como íconos a los más grandes delincuentes de los últimos tiempos como son Alberto Fujimori y Vladimiro Montesinos, que aunque parezca increíble hasta la fecha tiene seguidores y admiradores, algo totalmente fuera de lo normal en una sociedad sana.

Esto se da porque la corrupción en nuestro país es esencialmente cultural, pero con efectos económicos desastrosos, como por ejemplo que por culpa de la corrupción miles de personas se ven privadas de empleo, aumenta la pobreza y lo que más grave, es que es imposible que se pueda iniciar un proceso de desarrollo sostenido (1), ya que la corrupción socava las bases mismas de cualquier sociedad sumiéndola en el caos y la anomia como esta sucediendo en el Perú y en la Región Junín.

\section{MATERIAL Y MÉTODOS}

Los materiales empleados fueron los bibliográficos, los archivos periodísticos y las encuestas realizadas por los organismos que realizan las mediciones $y$ sondeos sobre corrupción como por ejemplo de Proética. En cuanto a la metodología, utilizamos el método científico, cuyos procedimientos utilizados fueron el deductivo y el inductivo. A esto se puede agregar el método histórico.

\section{RESULTADOS}

Comenzaremos con la percepción de la corrupción en el continente. La Comparación que se puede hacer en este caso es con los países vecinos. De todos ellos el más corrupto es Ecuador y el menos corrupto Chile (para el año 2000), con un índice de 2,6 y 7,4 respectivamente haciendo notar que cuanto más bajo es el índice, existe mayor corrupción. En tanto que Perú tiene 4,4 por encima del promedio entre los países limítrofes. La corrupción en el Perú viene desde muy antes, incluso desde la era Prehispánica (2). Se dice por ejemplo que en el incanato la corrupción ya era conocido, de allí la presencia de ciertos funcionarios llamados tukuy rikuy (que todo los ven y que todo lo oyen), como signo de coerción para combatir la corrupción y someter a la población a los dominios del Inca. Pero muchos de estos funcionarios caían en lo que comúnmente se llama coima y sus informes eran adulterados para favorecer a ciertos curacas, claro con el beneficio para el veedor, pero muchas veces esto era castigado salvajemente, con la amputación de los miembros superiores o la muerte. Todo esto revela que la corrupción pública era muy fuerte y de suma gravedad en esos tiempos. Asimismo, el Estado inca favoreció la práctica de una conducta patrimonial respecto al uso de los bienes públicos, alimentando la cosmovisión del funcionario público respecto a que el gobierno se debe manejar como un feudo personal (2), como se observa actualmente en algunos funcionarios. Luego vino la invasión Española, con todas sus secuelas de corrupción y distorsión de las costumbres y normas de convivencia (las instituciones) trastocando durante casi Quinientos años la vida de la sociedad Peruana, ya que las reglas de conducta que trajeron los españoles se mixtifico y se pervirtió en los primeros tiempos de la Colonia hasta parecer normal, por que los invasores eran de lo peor de la gente europea que habían Ilegado a estas tierras. Es decir, las personas que vinieron a estas tierras eran sujetos sumamente corruptos cuyas prácticas hasta ahora se puede ver de manera similar, sobre todo en la política, al caso del personaje Francisco de Carvajal, el famoso "Demonio de Los Andes"(3), cuyo comportamiento es hasta hoy referente para muchos políticos y funcionarios del Estado peruano. Pero es en estos últimos treinta años que la corrupción ha crecido enormemente, especialmente con la llegada al poder de la mafia fujimontesinista. Es consenso que los niveles de corrupción en el 
gobierno del delincuente Fujimori es el mas grande a todo lo largo y ancho de la historia peruana. Se ha llegado a calcular que la corrupción visible y cuantificable de este gobierno costó aproximadamente 872 millones de dólares, solamente de aquello que se tiene evidencia y cuyos implicados se encuentran presos o con orden de captura, pero no refleja el verdadero monto de la corrupción en general, ya que algunos calculan en cinco mil millones de dólares y otros en ocho mil millones, incluyendo lo estimado del robo realizado por el ex - presidente A. Fujimori (4). Por otro lado, una de las consecuencias de la corrupción en el Perú se da en el plano económico como se puede ver en las siguientes tablas:

Tabla 1. Costo de oportunidad de la corrupción.

\begin{tabular}{|c|c|c|}
\hline $\begin{array}{l}\text { Costo de oportunidad de la corrupción } \\
\text { para el país }\end{array}$ & $\begin{array}{l}\text { Aumento en el ratio } \\
\text { Imversión/ PBI }\end{array}$ & $\begin{array}{l}\text { Aumento \% on ol } \\
\text { PBI per cápita } \\
\text { anual }\end{array}$ \\
\hline 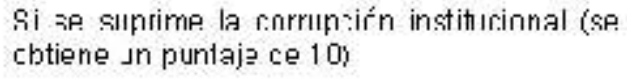 & De $2 ?, 6 \%$ a $.3 \angle, 6 \%$ & $\begin{array}{l}1,5 \% \\
\text { (US } \$ 637 \text { millcnes) }\end{array}$ \\
\hline $\begin{array}{l}\text { El mejora } 2 \text { furtos en el indice de percapsicn } \\
\text { de a sorrupción }\end{array}$ & ve $22, b \%$ a $2 t, b \%$ & $\begin{array}{l}\text { U, 5\% } \\
\text { (US4 } 258 \text { millcnes) }\end{array}$ \\
\hline $\begin{array}{l}\text { Si me ora funto en el İndice de se’cepción } \\
\text { de la corrupciór }\end{array}$ & $\mathrm{Ce} 226 \% 2246 \%$ & $\begin{array}{c}0.25 \% \\
\text { (UO\$ } 129 \text { millones) }\end{array}$ \\
\hline
\end{tabular}

Fuent: Waxo, Paolo (1395)

Es decir, si se elimina la corrupción se mejora los índices del producto bruto interno y significa crecimiento de la economía cuyos impactos se pueden ver sobre en las capas más pobres de la

Tabla 2. El costo económico anual de Ia corrupción Inerclal, regular.

\begin{tabular}{cl}
\hline Variable & \multicolumn{1}{c}{ Cifra } \\
\hline PBI & US $\$ 697$ millones \\
tnpleo & $62,6\lrcorner 1$ pJestos de t ajalo que no se jeneren \\
Pobresa & 57,570 personas que no dejan la pob'eza \\
\hline
\end{tabular}

Filenter riFSCO

población (ver la siguiente tabla).

Como se podrá observar, la corrupción es como un cáncer para la sociedad, ya que en el caso peruano, miles de personas se encuentran en pobreza a causa de la corrupción, y no es como algunos sofistas de la política lo pintan que los pobres son pobres porque quieren. Los recursos desviados hacia la corrupción pueden considerarse como una transferencia pública. En este caso los recursos se concentrarían en el sector de mayores ingresos, teniendo un comportamiento claramente regresivo sobre la distribución del ingreso. Este hecho no hace sino restringir el crecimiento económico del país y la recaudación de mayores impuestos. Los recursos de la corrupción acaban concentrándose -para su usufructo- en los sectores de mayores ingresos relativos, en desmedro de los sectores de menores ingresos que ven recortado el presupuesto público y por esta vía la calidad y cobertura de la inversión social que los beneficiaría directamente. Por eso algunos consideran que la corrupción constituye un impuesto altamente regresivo ya que no hace sino concentrar más los recursos en el decil de mayores ingresos. Para los sectores en pobreza extrema o desempleados, la corrupción les limita sus oportunidades de salir de la pobreza y de tener acceso al mercado de trabajo en condiciones dignas. Si la corrupción inercial ya significaba un costo importante para la economía: menos inversión, menos crecimiento, mayor desempleo y mayor pobreza; la presencia de la corrupción sistémica de la década anterior puede verse como el sobre costo económico de ella.

En cuanto a la corrupción en la región Junín, este es sumamente grave, ya que por ejemplo en la portada de una diario local (5), se escribía lo siguiente: "JUNIN CON 65\% DE CORRUPCIÓN", el sector EDUCACION: Con red enquistada por años. UNIVERSIDADES: Negocian notas, chantajes sexuales y acomodos de docentes. SALUD: Médicos trasladan a pacientes a consultorios y tráfico de medicinas. PODER JUDICIAL: Cobros por trámites y arreglo de sentencias. PNP: Robo de s c a rado de combustible y coimas de Policías de Tránsito. GOBIERNO REGIONAL: Pago de 8 mil soles por logotipo e irregularidades. Más adelante, dice que "Junín se pudre en la corrupción" donde el índice de percepción de la corrupción alcanza el $83 \%$. Esto está basado en un Estudio de Proética (6) que se presenta en la siguiente tabla, en el cual se muestra las instituciones según índice de percepción de corrupción:

Del cuadro anterior se desprende que la institución más corrupta es el poder judicial, que coincide con la percepción nacional, es decir, tanto a nivel nacional como regional, el ciudadano común y corriente percibe que la raíz de la corrupción se encuentra en 
el poder judicial. Esto significa que mientras la justicia no sea igualitaria para los miembros de una sociedad, ésta se tornara incierta y débil, al mismo tiempo que la articulación en los poderes del estado, la sociedad civil y las organizaciones no tengan un curso adecuado para superar la pobreza, el subdesarrollo y el atraso. Esto se puede observar todos los días y a todos los niveles en el caso del poder judicial. Ya que mientras exista una diferencia entre, los que han delinquido por necesidad extrema (hambre y necesidades básicas) sufren

Tabla 3 . Instituciones percibidas como las más corruptas en Junín y a nivel nacional (2004).

\begin{tabular}{|c|c|c|c|}
\hline \multirow[b]{2}{*}{ Orden } & \multirow[b]{2}{*}{ Institución } & \multicolumn{2}{|c|}{ Porcentaje de mencionee eepontáneae } \\
\hline & & Junín & Macional \\
\hline 1 & Poder undicial & 13 & 73 \\
\hline 2 & Hollcia Nacional & iU & bt \\
\hline 3 & Gobierno certral & 68 & 14 \\
\hline 4 & Congreso de la Resública & 64 & 25 \\
\hline 5 & Gobierno regional & 43 & \\
\hline 6 & Munic ifalidades & 37 & \\
\hline 7 & Partidos politicos & $\cdot 9$ & $1 \mathrm{c}$ \\
\hline 0 & F scalía de la Nacion & 7 & \\
\hline 9 & F Jerzas Armadas & 2 & $1 E$ \\
\hline \multirow{2}{*}{10} & Contraloría General de la Repúblic a & 9 & \\
\hline & Profescrado & 9 & \\
\hline
\end{tabular}

Fuente: Mapa de Riesgos de Corrukción Junín.

Elakoración propia

todo el peso de la ley, además que se encuentran hacinados en los diversos centros penitenciarios, los delincuentes de cuello y corbata que en su mayoría son funcionarios del Estado y empresarios corruptos que viven del Estado como fuente de riqueza son tratados benévolamente y es más hasta son apoyados por todos los poderes del Estado como la pretendida ley sobre arresto domiciliario, eso hace que nadie tome en seriedad las reglas básicas de la convivencia en sociedad y de allí la anomia y caos en que se encuentra la sociedad peruana. En otras palabras los ciudadanos perciben que mientras haya injusticia entonces no están obligados a respetar a nadie, salvo que se imponga la fuerza, coacción y otra forma de incentivo que les beneficie y si es fácil mejor (léase corrupto).

Asimismo, el Dr. Raúl Villavicencio (7), titular de la Procuraduría Anticorrupción de Junín, manifestó que la región es la capital de la corrupción, debida al elevado número de denuncias existentes. Mil 500 funcionarios públicos investigados por casos de corrupción. En el último mes (Junio del 2003) los casos denunciados aumentaron de 800 a 855 . El $94 \%$ de ellos se encuentra en proceso de instrucción en el Poder Judicial. En este aspecto se puede ver que en todos los estamentos de la administración pública se encuentran indicios 0 actos de corrupción. Es decir ningún estamento de la administración pública se encuentra libre de corrupción. Es más todos los alcaldes distritales tiene alguna denuncia o juicio por corrupción en el poder judicial. Pareciera que dichos funcionarios solo llegan al cargo para delinquir y esto con la venia de los pobladores, hasta se ha hecho costumbre y parece normal decir: "roba pero hace algo".

Lo anterior corrobora que a nivel nacional el departamento de Junín se encuentra en el pináculo de la corrupción y tristemente nos hacemos famosos. Esto se puede ver en la siguiente tabla que mide el índice de corrupción y de gobernabilidad a nivel público por departamentos. En esto debemos advertir lo tratado hasta ahora sólo es del sector público, porque lamentablemente no existen datos fiables para el sector privado, pero debemos decir que este sector también está articulado al proceso de corrupción.
Tabla 4. índice de corrupcion y gobernabildad (ICG) por departamento: 2004

\begin{tabular}{|c|c|c|c|}
\hline Departamento & índlce & Deparamento & Índlce \\
\hline 1. JJnin & $12,5 x$ & 1:. Lamoayeque & 548 \\
\hline 2. Ap Јгіmac: & $12,1 \%$ & 4. mad'e de clos & 44.6 \\
\hline 3. Pascc & $11,2 \%$ & 1. Uc arjali & 426 \\
\hline 2. La Libertad & $93 \%$ & 1E. Cuzco & 965 \\
\hline 5. Pบาด & $81 \%$ & 17. Ancagh & $35 \%$ \\
\hline 6. Lima Y . & $72 \%$ & 1E. Huancarelca & $35 \%$ \\
\hline Piurritadiu & $6.4 \%$ & 1द. Ljrelu & $31 \%$ \\
\hline i. Pilla & $63 \%$ & 2C. Moquegua & 238 \\
\hline 8.160 & $63 \%$ & 21. Tacra & $22 \%$ \\
\hline 9. Turbas & $61 \%$ & 2. Caarrarca & 208 \\
\hline 1ก HIİ̈nurn & $57 \%$ & arrarnnas & $19 \%$ \\
\hline 11. Af at:ul:lu & $56 \%$ & 24. Sall Ma lir & 098 \\
\hline 12 Arequisa & $55 \%$ & & \\
\hline Fuente: PRCET & & & \\
\hline
\end{tabular}

Como se puede observar, JUNIN es el departamento más corrupto del país, que tiene un índice de corrupción el doble del promedio nacional $(12,5 \%$ frente al $6,4 \%)$. Aunque, debemos señalar una 
observación en el sentido de que en el departamento de San Martín no creemos que sea posible tenga ese nivel ya que en dicho departamento se desarrolla uno de los conflictos sociales más graves del país como es el fenómeno del narcotráfico y que en cualquier parte del Mundo es uno de los elementos generadores de mayor corrupción en todo y más todavía en nuestro país que sumado a la pobreza hacen la trilogía del infierno en que viven sumidos los pueblos nororientales del país: corrupción, pobreza y narcotráfico.

En el siguiente cuadro se puede observar qué tipos de profesionales son los más corruptos en el desempeño de la función pública, haciendo hincapié que este resultado salió de un muestreo a personas con educación superior ya sean estudiantes o profesionales

Tabla 5. Índice de corrupción entre profesionales: Junín.

\begin{tabular}{cc}
\hline Profesionales & Índice \\
Juez & $86 \%$ \\
A bogado & $83 \%$ \\
Médico & $76 \%$ \\
Profesor de colegio & $65 \%$ \\
Policía & $63 \%$ \\
Arquitecto & $57 \%$ \\
Otros & $40 \%$ \\
\hline
\end{tabular}

Fuente: Encuesta realizada el mes de abril del 2005

Como se observa, el profesional del Derecho para la opinión pública es el más corrupto (83\%). Esto explica que, mientras el ciudadano común y corriente percibe que la justicia sea una mercancía, toda conducta se puede alterar y de todo lado se puede sacar provecho. En otras palabras se puede ser corrupto mientras hay justicia que comprar. Allí se encuentra una de las fuentes toda la corrupción en Junín y el Perú, que suma incorpora a la conducta y cultura del ciudadano.

\section{DISCUSION}

La corrupción tiene elementos culturales y económicos difícil de combatir sólo con medidas paliativas. El Perú es más corrupto que Chile y algunos países vecinos; esto puede ser tentador para futuras agresiones militares de los países vecinos; dado que un país corrupto es más vulnerable. Asimismo, Junín es el departamento más corrupto a nivel nacional, con $12,5 \%$ de índice de corrupción y gobernabilidad, por el cual es Ilamado "la capital de la corrupción". Todo esto se inserta dentro de un marco cultural y económico, por eso, para el ciudadano común y corriente el profesional del derecho es el profesional más corrupto y es la fuente de la corrupción en general, dado que dicho profesional recala principalmente en el poder judicial. Esto permite que la corrupción impida el inicio de todo proceso de desarrollo (1), ya que la corrupción genera pobreza, recesión y desempleo. O sea, existe una estrecha vinculación entre corrupción y desarrollo. Dicha relación es indirecta; mientras menos corrupto sea una sociedad más desarrollo tendrá y será más fácil salir del subdesarrollo.

Por otro lado, la corrupción en el Perú y por lo mismo en Junín viene desde los tiempos prehispánicos (2) y está asociado fuertemente a un componente cultural que se conoce como la reciprocidad andina fuente y origen de la corrupción en el país y que fue agravado con la invasión española, añadiendo el concepto de viveza criolla que también es fuente de corrupción. Son estos dos elementos factores esenciales de la corrupción en Junín a nivel cultural.

A nivel económico, la pobreza y las escasas oportunidades de los agentes económicos es la fuente de la corrupción.

\section{REFERENCIAS BIBLIOGRÁFICAS}

1. Programa de las Naciones Unidas para el Desarrollo. Informe sobre democracia e índice de libertad). Citado en Abril del 2005. En http://www.pnud.org.pe

2. Vargas Llosa, Álvaro. La contenta barbarie. España: 1ra. Edición; 1993.

3. Palma, Ricardo. Tradiciones Peruanas. Lima: Primera edición; 1976.

4. La Gaceta Parlamentaria. Informe sobre la Corrupción. La República 2002. Marzo 24, Página 6. (Revista del Congreso de la República del Perú)

5. Diario PRIMICIA: Página de portada. 2005 Abril página 15.

6. PROETICA. Consejo Nacional para la Ética Pública. "Mapa de Riesgos de la Corrupción".Citado en noviembre del 2004. En: http://www.proetica.org.pe/index.php

7. Diario Correo. Corrupción en Junín. Declara Procurador anticorrupción. 2004. Junio 10; página 4.

E-mail: dardm007@hotmail.com 